\title{
Laser Guide Stars for Optical Free-Space Communications
}

\author{
Ramon Mata Calvo*a ${ }^{\mathrm{a}}$, Domenico Bonaccini Calia ${ }^{\mathrm{b}}$, Ricardo Barrios ${ }^{\mathrm{a}}$, \\ Mauro Centrone $^{\mathrm{c}}$, Dirk Giggenbach ${ }^{\mathrm{a}}$, Gianluca Lombardi ${ }^{\mathrm{d}}$, Peter Becker ${ }^{\mathrm{a}}$, Igor Zayer ${ }^{\mathrm{e}}$ \\ ${ }^{\mathrm{a}}$ German Aerospace Center, Oberpfaffenhofen, Münchener Straße 20, 82234, Germany; \\ ${ }^{\mathrm{b}}$ European Southern Obserbatory, Karl-Schwarzschild-Str. 2, 85748 Garching, Germany; \\ 'INAF - Osservatorio Astronomico di Roma, Via di Frascati 33, 00040 Monteporzio Catone, Italy; \\ ${ }^{\mathrm{d}}$ GRANTECAN S.A. Cuesta de San José, s/n -38712 - Breña Baja - La Palma, España \\ ${ }^{\mathrm{e}}$ ESA-ESOC, Robert-Bosch-Strasse 5, 64293 Darmstadt, Germany
}

\begin{abstract}
The German Aerospace Center (DLR) and the European Southern Observatory (ESO) performed a measurement campaign together in April and July 2016 at Teide-Observatory (Tenerife), with the support of the European Space Agency (ESA), to investigate the use of laser guide stars (LGS) in ground to space optical communications.

Atmospheric turbulence causes strong signal fluctuations in the uplink, due to scintillation and beam wander. In space communications, the use of the downlink channel as reference for pointing and for pre-distortion adaptive optics is limited by the size of the isokinetic and isoplanatic angle in relation to the required point-ahead angle. Pointing and phase errors due to the decorrelation between downward and upward beam due to the point-ahead angle may have a severe impact on the required transmit power and the stability of the communications link.

LGSs provide a self-tailored reference to any optical ground-to-space link, independently of turbulence conditions and required point-ahead angle. In photon-starved links, typically in deep-space scenarios, LGSs allow dedicating all downlink received signal to communications purposes, increasing the available link margin.

The scope of the joint DLR-ESO measurement campaign was, first, to measure the absolute value of the beam wander (uplink-tilt) using a LGS, taking a natural star as a reference, and, second, to characterize the decrease of correlation between uplink-tilt and downlink-tilt with respect to the angular separation between both sources. This paper describes the experiments performed during the measurement campaigns, providing an overview of the measured data and the first outcomes of the data post-processing.
\end{abstract}

Keywords: Laser Guide Star, Ground-to-Space, Free-Space Optical Communications, Beam Wander, Isokinetic angle, Uplink Channel, Laser Communications

\section{INTRODUCTION}

Laser guide star (LGS) technology enables adaptive optics for large ground-based telescopes because it can provide a sufficiently bright reference anywhere in the sky ${ }^{1-4}$. The turbulence in the lower part of the atmosphere $(0-20 \mathrm{~km})$ distorts the incoming light from a science object. The adaptive optics system can use the LGS reference close to the science object to correct the atmospheric aberrations even for very faint science objects and without needing any bright star close to it. The collaboration between DLR and ESO targets the potential use of LGS in optical communications, where a pre-distortion of the uplink signal would enhance the performance of satellite communications based on optical technologies.

Optical communications to space are promising because of the available bandwidth, the lack of spectrum regulations, and the smaller size and power requirements. Since the nineties, optical communication in space has been growing, with the first demonstration of optical inter-satellite links between low-earth orbit (LEO) satellites and geostationary orbit

* Ramon.MataCalvo@dlr.de; phone +49 815328 3448; fax +49 8153 28-2844

Copyright 2017 Society of Photo-Optical Instrumentation Engineers. This paper was published in SPIE Photonics West 2017 and is made available as an electronic reprint with permission of SPIE. One print or electronic copy may be made for personal use only. Systematic reproduction and distribution, duplication of any material in this paper for a fee or for commercial purposes, or modification of the content of the paper are prohibited. 
(GEO) satellites from SPOT-4 and Artemis ${ }^{5}$. This demonstration was the first step towards the European Data Relay System (EDRS) ${ }^{6}$ which currently is being deployed. Further demonstrations followed from LEO and GEO to ground links ${ }^{7-9}$ showing the feasibility of such technology in space. In 2014 with the links to the spacecraft LADEE optical technologies allowed data exchange between the probe orbiting the Moon and the Earth ${ }^{10}$.

An uplink signal from the Earth towards the space is used for data transfer like in GEO Feeder-links or as reference for a spacecraft in deep-space communications, to improve the power efficiency and communications performance. When sending a laser beam from ground to space, the atmospheric turbulence introduces an uplink-tilt which produces a beam wandering at the receiver side. At the spacecraft, the beam-wander or uplink-tilt has a high impact in the signal fluctuations, causing strong penalties, often referred as "fading". This is critical in GEO feeder-links due to the high throughputs where a fade means the loss of millions of bits. In deep-space scenarios, the power efficiency is crucial to be able to reach larger distances.

Usually in satellite communication, the downlink signal is used as reference for pointing the uplink. But this works only whenever the point-ahead-angle is smaller than the isokinetic angle; i.e. the coherent area where the atmospheric-induced tilt can be assumed to remain constant. When the point-ahead angle is too large, the divergence of the uplink beam is increased to cope with the beam-wander fluctuation, this however has an impact in the power link-budget, which is critical in both GEO feeder-links and deep-space communications.

LGS are used in astronomy for applying adaptive optics when pointing towards faint objects. Such a reference has not been used in satellite communications yet. Particularly, how a LGS can help in compensating the uplink-tilt, which is the effect that has a higher impact in the uplink signal stability and communications performance.

The objective of this paper is to present the preliminary results of the measurements performed with a LGS in April and July 2016, thanks to collaboration between DLR, ESO and with the support of ESA. First the theoretical description and the experimental setup are presented. The preliminary results of the measurements of beam wander (uplink-tilt) and correlation between downlink paths with respect to their angular separation are showed.

\section{UPLINK TILT MEASUREMENT FROM LASER GUIDE STAR}

\subsection{Beam-wander, point-ahead angle and tilt-isoplanatic angle}

Uplink-tilt causes random fluctuations of the beam pointing direction, due to the refraction of the turbulence cells that are large compared to the laser beam radius. Beam wander (or uplink-tilt) contributes to the beam spread in the longterm.

$$
\sqrt{\left\langle\alpha_{B W}^{2}\right\rangle}=0.73\left(\frac{\lambda}{2 W_{0}}\right)\left(\frac{2 W_{0}}{r_{0}}\right)^{5 / 6}
$$

The uplink-tilt standard deviation $\sqrt{\left\langle\alpha_{B W}^{2}\right\rangle}$ depends on the wavelength $\lambda$, the transmitted beam radius $W_{0}$ and the Fried parameter $r_{0}$.

The point-ahead angle is needed for compensating the satellite movement during the light transmission time between the ground station and the space probe. It can be seen as the angular separation between the uplink and the downlink. The point-ahead angle ranges between few micro-radians up to hundreds micro-radians, depending on the satellite or spacecraft velocity. Ideally if the point-ahead angle becomes zero, all aberrations introduced by the turbulence in the uplink could be estimated using the downlink. As the point-ahead angle increases, the correlation of the atmospheric effects between downlink and uplink decreases.

The isoplanatic angle defines the angular separation of two directions in which one can assume the atmospheric wavefront distortions are still correlated, i.e. the mean-squared wave-front error between them is $1 \mathrm{rad}^{2}$. The isoplanatic angle

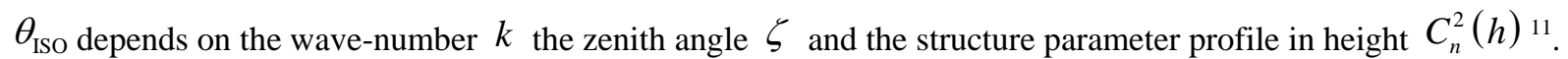

$$
\theta_{\text {ISO }}=\left[2.914 k^{2} \sec (\zeta)^{8 / 3} \int_{0}^{\infty} C_{n}^{2}(h) h^{5 / 3} d h\right]^{-3 / 5}
$$


The isoplanatic angle takes all aberration orders into account. Sasiela ${ }^{12}$ (among others) studied the change of the isoplanatic angle with respect to the aberration order using the Zernike modes representation, leading to a tilt simplified formula for characterizing the tilt anisoplanatism. The tilt-isoplanatic angle or isokinetic angle $\theta_{\text {T-Iso }}$ is calculated as follows:

$$
\theta_{\mathrm{T}-\mathrm{ISO}}=\frac{0.184 \lambda\left(\sqrt{8} W_{0}\right)^{1 / 6}}{\left(\sec ^{3}(\zeta) \int_{h} C_{n}^{2}(h) h^{2} d h\right)^{1 / 2}}
$$
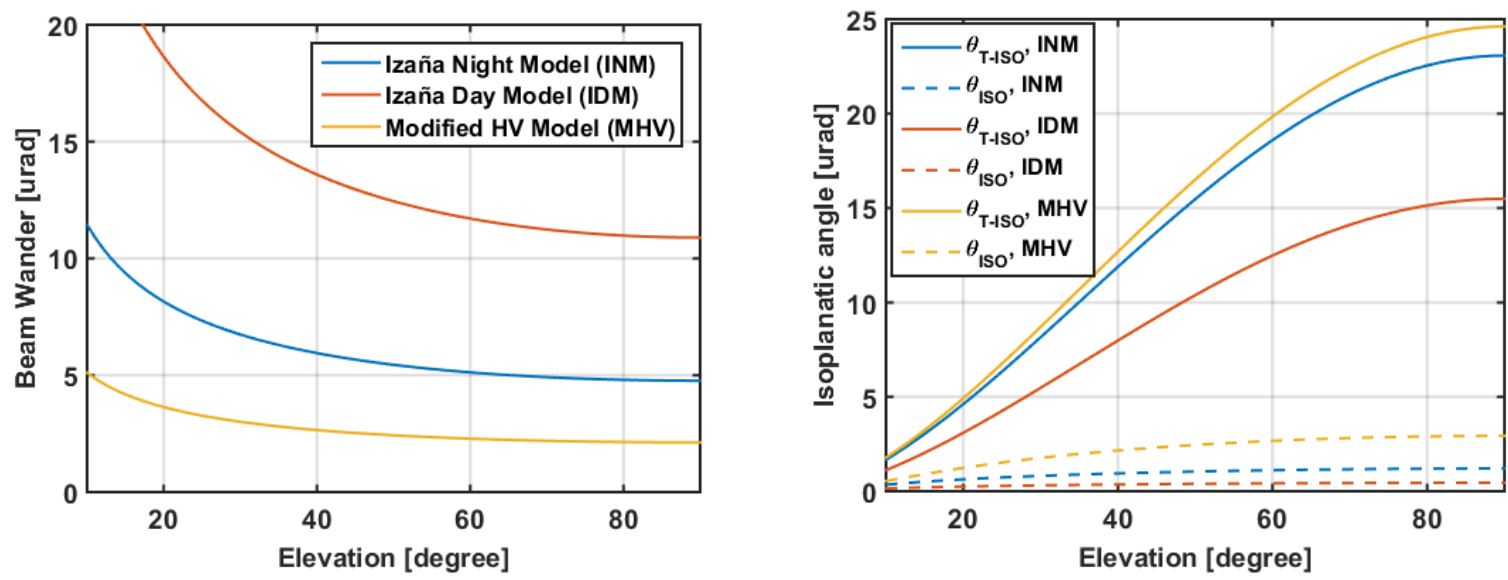

Figure 1. Left: Beam-Wander standard deviation over the elevation angle. Right: Isoplanatic angle and isokinetic angle over the elevation angle

In the left plot of Figure 1, the uplink-tilt is plot versus the elevation angle and in the right plot the isoplanatic angle and isokinetic angle $\theta_{\text {T-Iso }}$. In these theoretical calculations three models were used for the profile of the structure parameter in height $C_{n}^{2}(h)$ : the empirical models ${ }^{13}$ Izaña-Night (INM) and Izaña-Day (IDM) and a modified version of the Hufnagel-Valley (MHV) ${ }^{7}$. The wavelength was assumed $589 \mathrm{~nm}$ and the transmitter aperture of the LGS was assumed $20 \mathrm{~cm}$.

\subsection{Uplink tilt calculation}

The LGS can provide a reference of the phase distortions introduced by the atmosphere in the uplink. The backscattered light of the atmosphere's sodium layer is used to measure the phase distortions and apply a pre-distortion to the uplink signal. However, if the same aperture is used for transmitting the LGS and measuring the backscattered light, the LGS provides only information about the wave-front aberrations, but it is unable to determine the uplink-tilt, because the light is travelling through the same path twice, within the tilt coherence time of the atmosphere.

For measuring the uplink-tilt we use the method proposed by Ragazzoni and Esposito ${ }^{14,15}$ : using two separate locations, one for transmitting and one for measuring the LGS. The scope of this method was to estimate the downlink-tilt of the observing object using the LGS as reference and using a star as natural guide star (NGS) for estimating the LGS uplinktilt. In this case the calculation is simpler since we are interested only in the uplink-tilt of the LGS. The Launch Station (LS) sends the LGS and some meters a part the Observer Station (OS) receives light scattered by the sodium layer. Due to the parallax effect, the OS sees an elongated version of the LGS, a kind of "cigar" shape. The LGS pointing from the LS is adjusted to find a NGS in the OS field-of-view.

The OS can measure two tilts: the LGS tilt $\tilde{T}_{L G S}$ and the NGS tilt $\tilde{T}_{N G S}$ :

$$
\tilde{T}_{N G S}=T_{N G S}^{d}+J_{O S}
$$




$$
\tilde{T}_{L G S}=J_{L S}+T_{L G S}^{u}+T_{L G S}^{d}+J_{O S}
$$

The measured NGS tilt $\tilde{T}_{N G S}$ consists in the downlink-tilt of the NGS $T_{N G S}^{d}$ plus any jitter produced by the OS $J_{\text {OS }}$. The measured tilt from the LGS $\tilde{T}_{L G S}$ is the addition of the OS jitter, the LS jitter $J_{L S}$, the LGS uplink-tilt $T_{L G S}^{u}$ and the downlink-tilt of the LGS $T_{L G S}^{d}$. The $T_{L G S}^{d}$ can vary along the "cigar", depending on the size of the isokinetic patch. Due to the LGS elongation, the $\tilde{T}_{L G S}$ can be only measured accurately in one-axis, the one orthogonal to the LGS "cigar". Therefore only one-axis tilts are considered. Furthermore any noise sources are not taken into consideration:

By subtracting both equations, the uplink-tilt of the LGS can be calculated, depending on the correlation between both LGS and NGS downlink paths.

$$
\tilde{T}_{L G S}-\tilde{T}_{N G S}=T_{L G S}^{u}+T_{L G S}^{d}-T_{N G S}^{d}+J_{L S}
$$

If the NGS is located within the isokinetic patch of the LGS, then the term $T_{L G S}^{d}-\tilde{T}_{N G S}$ should tend to zero. Assuming $J_{L S}$ is negligible and the one-axis tilts are normal-distributed, the variance of the tilt subtraction can be calculated as follows, where $\operatorname{var}(\cdot)$ and $\operatorname{std}(\cdot)$ are the variance and standard deviation operators respectively.

$$
\operatorname{var}\left(\tilde{T}_{L G S}-\tilde{T}_{N G S}\right)=\operatorname{var}\left(\tilde{T}_{L G S}\right)+\operatorname{var}\left(\tilde{T}_{N G S}\right)-2 \rho_{L G S, N G S} \cdot \operatorname{std}\left(\tilde{T}_{N G S}\right) \cdot \operatorname{std}\left(\tilde{T}_{L G S}\right)
$$

The dependency between both random variables is taken into account by the correlation between the LGS and the NGS $\rho_{L G S, N G S}$. From Equation (1), the LGS is the addition of two tilts, an uplink and a downlink tilt, which are assumed independent random variables because the LGS is travelling through different atmospheric paths in uplink and downlink. The correlation between the LGS and NGS downlink paths $\rho_{L_{G S} \text {,NGS }}$ determines the limit of the accuracy in the uplinktilt estimation through the subtraction of both measured LGS and NGS tilts. It can be calculated from the measured variables as follow:

$$
\begin{gathered}
\operatorname{var}\left(T_{L G S}^{u}+T_{L G S}^{d}-\tilde{T}_{N G S}\right)=\operatorname{var}\left(T_{L G S}^{u}\right)+\operatorname{var}\left(T_{L G S}^{d}\right)+\operatorname{var}\left(\tilde{T}_{N G S}\right)-2 \rho_{L G S_{d}, N G S} \cdot \operatorname{std}\left(T_{L G S}^{d}\right) \cdot \operatorname{std}\left(\tilde{T}_{N G S}\right) \\
\operatorname{var}\left(T_{L G S}^{u}+T_{L G S}^{d}-\tilde{T}_{N G S}\right)=\operatorname{var}\left(\tilde{T}_{L G S}\right)+\operatorname{var}\left(\tilde{T}_{N G S}\right)-2 \rho_{L G S_{d}, N G S} \cdot \operatorname{std}\left(T_{L G S}^{d}\right) \cdot \operatorname{std}\left(\tilde{T}_{N G S}\right)
\end{gathered}
$$

The variance of $T_{L G S}^{d}$, the LGS downlink tilt, cannot be directly estimated from the measured tilt $\tilde{T}_{L G S}$, which contains the combined effect of both uplink and downlink tilts.

\section{EXPERIMENTAL VERIFICATION}

\subsection{Measurement campaign}

The European Southern Observatory (ESO), together with Instituto de Astrofísica de Canarias (IAC) is currently testing in the Canary Islands the extremely large telescope LGS-AO configurations using the $20 \mathrm{~W}$ CW, $589 \mathrm{~nm}$ laser unit of the ESO-Wendelstein Laser Guide Star Unit (WLGSU) ${ }^{2,3,16}$. Until July 2016, the WLGSU was located at the Observatorio de El Teide (Izaña, Tenerife Island), a few hundred meters apart from the ESA OGS 1m telescope. The availability from ESO of an experimental, deployable WLGSU and its location offered a unique opportunity of studying the applicability of LGS for the uplink beacon in optical communications, fostering the first feasibility tests and starting a scientific collaboration between ESO and DLR in that field, with the support of ESA. Two measurement campaigns have been performed in 2016. In Figure 2, the left picture shows the ESO-LS with the LGS turned on. 

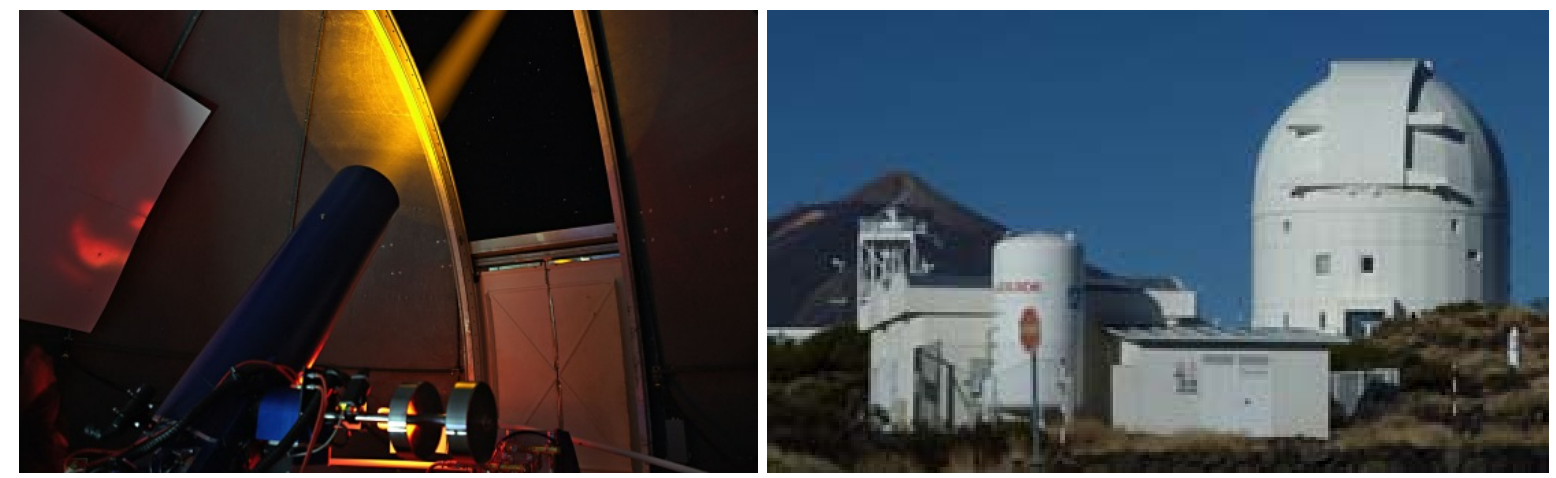

Figure 2. Left: ESO LGS mounted in Tenerife. Right: ESA optical ground station

The Observatorio de El Teide is located at $2370 \mathrm{~m}$ altitude above the sea level having multiple research facilities, among them the ESA OGS. The ESA OGS has performed optical communications links to satellites, from beginnings of 2000 with Artemis satellite within the SILEX experiment ${ }^{5}$ and currently to EDRS system satellites: Alphasat and EDRS-A ${ }^{17}$, see right picture in Figure 2. The ESA-OGS is equipped with a $1 \mathrm{~m}$ diameter telescope. Attached and aligned to the main telescope, a $20 \mathrm{~cm}$ guider telescope is also available. The ESA-OGS is about $110 \mathrm{~m}$ away from the ESO laser launch telescope. Moreover, at 11m distance from the ESO laser launch telescope (LS), an ESO $40 \mathrm{~cm}$ receiver telescope (OS) is installed and equipped to do automatic photometry of the LGS.

On using the second slit of the receiver NTM-500 Alt-Az mount, an extra telescope of $40 \mathrm{~cm}$ diameter was installed from DLR and co-aligned to the ESO receiver telescope. The DLR-OTA (Optical Tube Assembly) is a $40 \mathrm{~cm}$ Meade telescope. The final setup of the DLR- and ESO-OTA telescopes in the NTM-500 Alt-Az mount can be seen in the left picture in Figure 3.
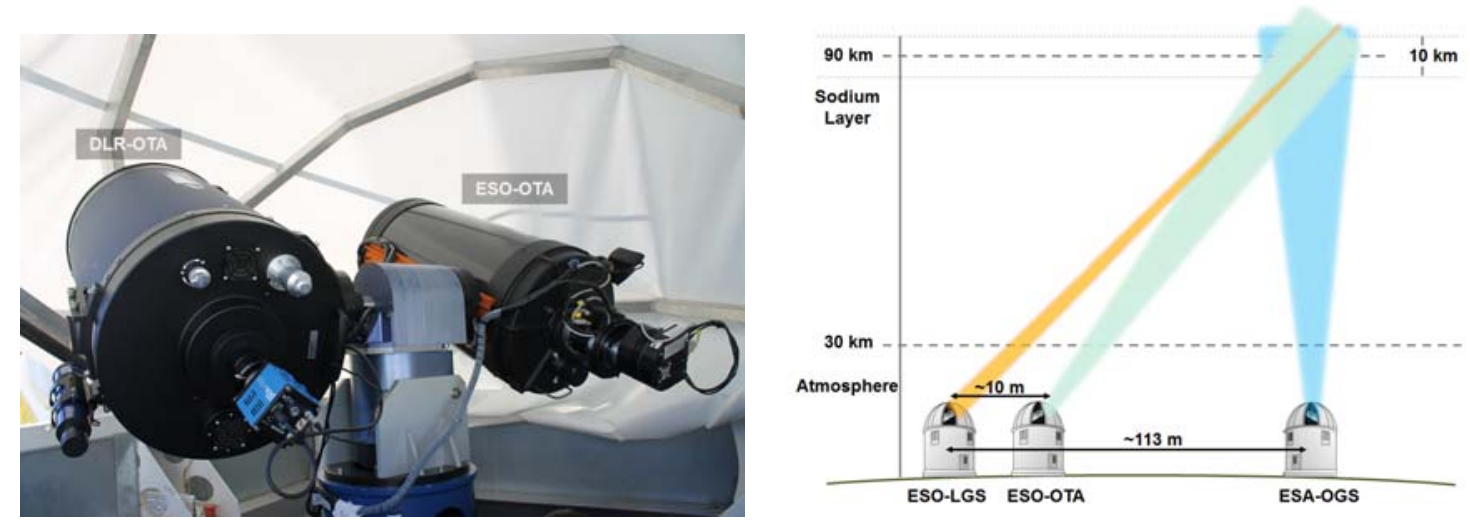

Figure 3. Left: $40 \mathrm{~cm}$ ESO-OTA and $40 \mathrm{~cm}$ DLR-OTA, mounted on an Astelco NTM-500 mount in Alt-Az configuration, centered 11 meters apart from the laser launch telescope. Right: measurement configuration description with two OS: the ESA-OGS and the DLROTA.

In Figure 3, the right picture shows the measurement configuration for the LGS observation with two OS: the ESA-OGS and the DLR-OTA at the ESO photometry receiver telescope station. The sodium layer is at $90 \mathrm{~km}$ altitude with around $10 \mathrm{~km}$ FWHM thickness. Because of the larger distance between the LS and the ESA-OGS, the observed LGS at the ESA-OGS is much more elongated than at the DLR-OTA.

Thus two telescopes were available and have been used for our measurements: the 1 meter telescope at the ESA-OGS and the $40 \mathrm{~cm}$ telescope DLR-OTA at the ESO photometry receiver station. The $20 \mathrm{~cm}$ guider telescope was used to get a large field-of-view image to facilitate the localization of the LGS seen at the 1 meter Cassegrain telescope focus. 


\subsection{Link budget calculation and requirements}

The expected number of photons per pixel was estimated assuming that the LGS produces a photon flux of $\sim 15 \mathrm{MPhotons} / \mathrm{m}^{2} / \mathrm{s}$, which corresponds to a star with visual apparent magnitude seven ${ }^{18}$. Additional assumptions are an integration time of $10 \mathrm{~ms}$ and a Fried parameter of $10 \mathrm{~cm}$.

The focal spot at both telescopes is limited by turbulence since the Fried-parameter is expected to be smaller than the aperture diameter. This leads to a much larger spot at the $1 \mathrm{~m}$ telescope due to the long focal length, which mostly offsets the benefit of the larger aperture area. The difference between the average photons per $\mu^{2}$ on the CCD sensor, for both telescopes, is therefore not significant although the photon flux is higher for the $1 \mathrm{~m}$ telescope.

Two cameras were identified to meet the low read-out noise requirements for short time exposures; their main characteristics are summarized in Table 1. During the measurement campaign in April, the pcoEdge 4.2 was used in the ESA-OGS and in July in the DLR-OTA. The iXon Ultra 888 was used in the ESA-OGS during the measurement campaign in July. In order to reject as much background light as possible and improve SNR an astronomical Johnson filter in the R band was placed in front of the camera. This type of filter has high transmission at around $590 \mathrm{~nm}$ and a bandwidth of about $100 \mathrm{~nm}$.

Table 1. Cameras used during the measurement campaign

\begin{tabular}{|l|l|l|}
\hline Parameter & pcoEdge 4.2 & iXon Ultra 888 \\
\hline Technology & sCMOS & EMCCD \\
\hline Quantum efficiency at 589nm [\%] & 70 & 95 \\
\hline Pixel size $[\mu \mathrm{m}]$ & 6.5 & 13 \\
\hline Read out noise $[\mathrm{e}-]$ & $<0.3$ & $<1$ \\
\hline Dark current $[\mathrm{e}-\mathrm{pixel} / \mathrm{s}]$ & $<0.3$ & 0.005 \\
\hline
\end{tabular}

Therefore, three receiver configurations can be defined for each telescope and camera. They are summarized in Table 2.

Table 2. Receiver configurations for both measurement campaigns

\begin{tabular}{|l|l|l|l|}
\hline Configuration & Telescope & Camera & Campaign \\
\hline RX-setup A & ESA-OGS & pcoEdge 4.2 & April \\
\hline RX-setup B & ESA-OGS & iXon Ultra 888 & July \\
\hline RX-setup C & DLR-OTA & pcoEdge 4.2 & July \\
\hline
\end{tabular}

As requirement for the post-processing of the focal images to be acquired, it was established that the recorded data shall have a minimum signal-to-noise ratio (SNR) of about ten, i.e. $~ 10 \mathrm{~dB}$. The calculation of the SNR per pixel of camera is given by

$$
S N R=\frac{P Q_{e} t}{\sqrt{P Q_{e} t+D t+N_{r}^{2}}}
$$

where $P$ is the photon flux, $Q_{e}$ is the quantum efficiency, $t$ is the integration time, $D$ is the dark current and $N_{r}$ is the read out noise. Figure 4 presents a plot with the SNR calculation of the different configurations of receiving telescope plus focal camera as listed in Table 2. In case the Fried parameter is $10 \mathrm{~cm}$ at $589 \mathrm{~nm}$, as it is assumed, the minimum integration time that achieves a SNR of 10 in RX-setup A is about $20 \mathrm{~ms}$, while for Setup B and C the integration time is about 5 and $10 \mathrm{~ms}$, respectively. The minimum integration time can reach 80-100 ms in a high turbulence condition, where a Fried parameter of $5 \mathrm{~cm}$ is assumed. The SNR equation (3) does not take any sky background noise contributions into account; the actual performance of the RX-Setups can differ from the results presented in Figure 4 . The minimum integration time, to produce focal images with sufficient SNR, is estimated to be in the order of $20 \mathrm{~ms}$ under moderate turbulence conditions. 

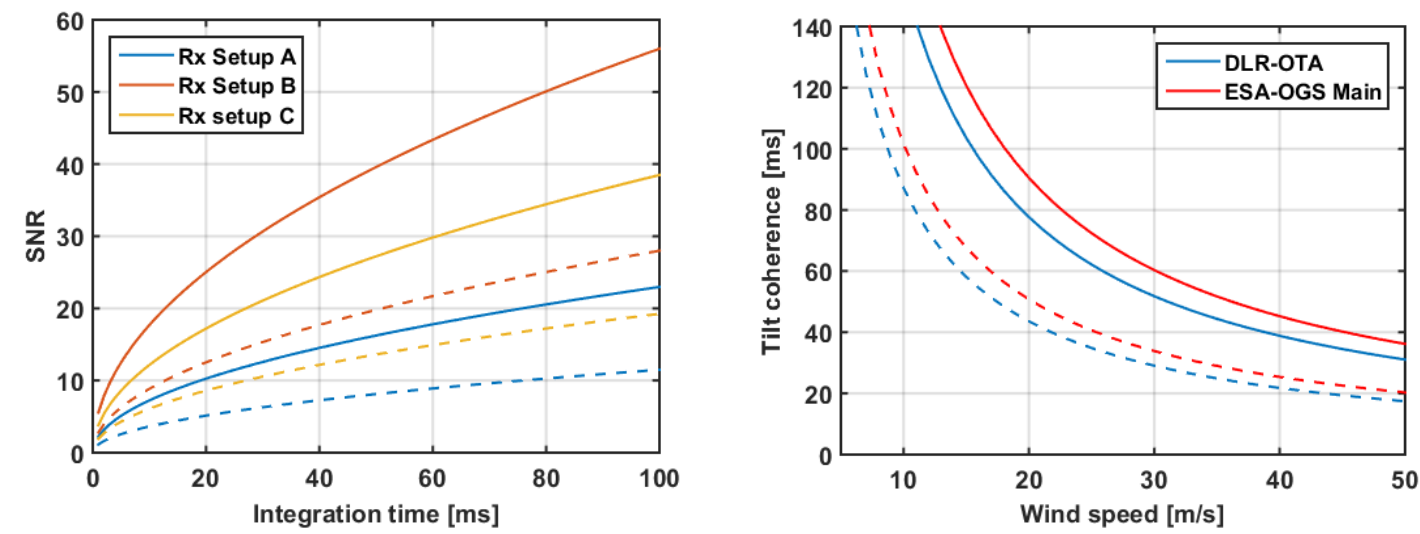

Figure 4. Left: SNR per pixel for all available configurations of telescope plus focal camera. The Fired parameter assumed was $10 \mathrm{~cm}$ (solid lines) and $5 \mathrm{~cm}$ (dashed lines). Right: Tilt coherence time vs wind speed for the two telescopes available for the measurement campaign. The Fired parameter assumed is $10 \mathrm{~cm}$ (solid lines) and $5 \mathrm{~cm}$ (dashed lines).

The integration time of the imager device was adjusted during the measurement campaign to achieve a focal image with a sufficient SNR for post-processing, by visual inspection of the recorded images. Nevertheless, the fundamental limitation of the integration time is given by the coherence time of the tilt. The tilt coherence time $\tau_{0}$ can be calculated as follows ${ }^{19}$.

$$
\tau_{0}=12.33\left(\frac{D}{r_{0}}\right)^{1 / 6}\left(\frac{r_{0}}{v}\right)
$$

where $D$ is the receiving telescope aperture, $r_{0}$ is the Fried parameter and $v$ is the wind speed transversal to the propagation of the laser beam. The tilt coherence time is plot in the right plot of Figure 4 for the ESA-OGS and the DLROTA telescopes. Assuming usual operation in wind speed conditions under $20 \mathrm{~m} / \mathrm{s}$, the tilt coherence time is in the order of hundreds of milliseconds or above. Thus, for integration times below $20 \mathrm{~ms}$, the tilt coherence time constraint should have been maintained.

The measurement campaign of April took place between the 11.04.2016 and the 19.04.2016. During this time, only the receiver telescope at the ESA-OGS was used. The measurement campaign of July took place between the 05.07.2016 and the 13.07.2016. During this time, both ESA-OGS and the DLR-OTA receiver telescopes were available, thus observing the same scene from two different positions at the same time was possible. As the observing station setups were not synchronized, instantaneous parameters cannot be directly compared for both sites, but the long term statistics should allow comparison.

\subsection{Available data and processing}

In Table 3 all measurements taken during both campaigns (April and July 2016) are summarized. In the first column is the name of the star targeted by the observing telescope, i.e. the star which the telescope was tracking. The reference time in the second column means the time at which the first sequence starts. In the third and fourth column there is the number of consecutive sequences for each target star and observation telescope: the ESA-OGS and the DLR-OTA. Each sequence contains from 4000 to 10000 images. The integration time was limited to $20 \mathrm{~ms}$, reaching 5 and $10 \mathrm{~ms}$ in some sequences, as expected from SNR estimations.

The raw data post-processing of the focal image is performed in different steps. First, data is inspected by a human operator to estimate a suitable size to crop the original image size down to the minimum size that would allow image analysis while reducing processing time. After this operation, the rest of the algorithm runs unsupervised to detect the different features of interest, i.e. the NGS and the elongated LGS. 
Table 3. List of target stars with the number of consecutive sequences for each observation station

\begin{tabular}{|c|c|c|c|c|c|c|c|}
\hline Target star & Reference time & OGS & OTA & Target star & Reference time & OGS & OTA \\
\hline Procyon & 11.04.2016 23:33:58 & 1 & & HIP76088 & 17.04.2016 02:27:42 & 8 & \\
\hline Procyon & 12.04.2016 21:51:24 & 1 & & HIP57706 & 18.04.2016 23:00:52 & 22 & \\
\hline Regulus & 12.04.2016 22:08:22 & 1 & & HIP76088 & 19.04.2016 00:55:39 & 18 & \\
\hline HIP67579 & 12.04.2016 22:31:17 & 7 & & & & & \\
\hline HIP56382 & 12.04.2016 23:42:26 & 4 & & HIP73286 & $07.07 .2016 \quad 22.21 .25$ & 4 & \\
\hline HIP58335 & 13.04.2016 01:25:25 & 2 & & HIP93321 & $07.07 .2016 \quad 00.57 .08$ & 2 & \\
\hline HIP68535 & 13.04.2016 02:21:42 & 3 & & HIP109 & 10.07.2016 06:06 & & 6 \\
\hline Sirius & 13.04.2016 21:15:00 & 1 & & HIP109366 & 10.07.2016 04.38.18 & 8 & \\
\hline HIP63900 & 13.04.2016 22:07:20 & 8 & & HIP73286 & 10.07.2016 22.14.26 & 1 & \\
\hline HIP67579 & 13.04.2016 20:50:54 & 1 & & HIP76972 & 10.07.2016 22.32.15 & 5 & \\
\hline HIP63900 & 13.04.2016 23:38:18 & 2 & & HIP92399 & 11.07 .201600 .05 .15 & 14 & 5 \\
\hline Mizar & 14.04.2016 22:55:51 & 1 & & HIP109366 & 11.07.2016 03:50 & & 15 \\
\hline Spica & 14.04.2016 21:02:18 & 1 & & HIP99502 & 11.07.2016 07:04 & & 7 \\
\hline HIP61385 & 15.04.2016 21:15:19 & 38 & & HIP2208 & 12.07.2016 03.59.20 & 12 & \\
\hline HIP51027 & 16.04.2016 23:31:21 & 2 & & HIP13941 & 12.07.2016 05.26.13 & 6 & 1 \\
\hline HIP57706 & 16.04.2016 23:56:28 & 18 & & HIP115185 & 12.07.2016 02.56.17 & 5 & 8 \\
\hline HIP74409 & 17.04.2016 01:53:53 & 1 & & SAO121174 & 12.07.2016 22.28.09 & 13 & \\
\hline
\end{tabular}

The image processing algorithm first performs a de-noising of the image; the SNR conditions of the scene were intrinsically low. Various types of noise reduction numerical filter were tested. Particular care was taken in selecting the filter, to avoid distorting the original image shape and above all avoid modifying the energy spatial distribution across the focal image plane. As the main information to be extracted is positional, there was a strong limitation in selecting a suitable filter: some filters had an influence on the spatial position of the peak pixel value in the order of 1-2 pixels. Among the tested numerical filters there are a 2D Gaussian convolutional filter, a median filter and a wavelet filter using different kernels. Finally, the selected filter was a wavelet filter with a Symlet kernel of 4 coefficients.

After filtering, to find the two elements of interest, i.e. the LGS and the NGS, morphological operations are applied to the filtered image. First, the NGS is detected by applying a threshold to the full image, because during the experiments the NGS always had larger SNR than the LGS. The threshold $\tau$ is automatically selected based on the following criteria

$$
\tau=\mu+n \cdot \sigma
$$

where $\mu$ and $\sigma$ are the mean and standard deviation of all the image pixels, and $n$ is a factor that helps to discriminate signal pixels from noise pixels. The value of $n$ was selected automatically between 2 and 5 based on the SNR of the image being processed. The thresholding operation produces a black and white image, which is further analyzed to detect a circular area of a minimum radius that corresponds to NGS, through the use of a Hough transform. This helps to identify the position of the NGS in the overall image space, while allowing masking out the NGS pixels, contributing to a better detection of the LGS pixels in the subsequent step.

After masking out the NGS, a new threshold is estimated using (5), to detect the LGS pixels and a median filter is applied to the new black and white image. This allows eliminating high value noise pixels, which are more likely to be found in the LGS detection due to the inherent low SNR of the signal. Next, a convex hull image is generated to detect the edges and area of the LGS. Finally, the inclination of the major axis of the LGS cigar is estimated. 

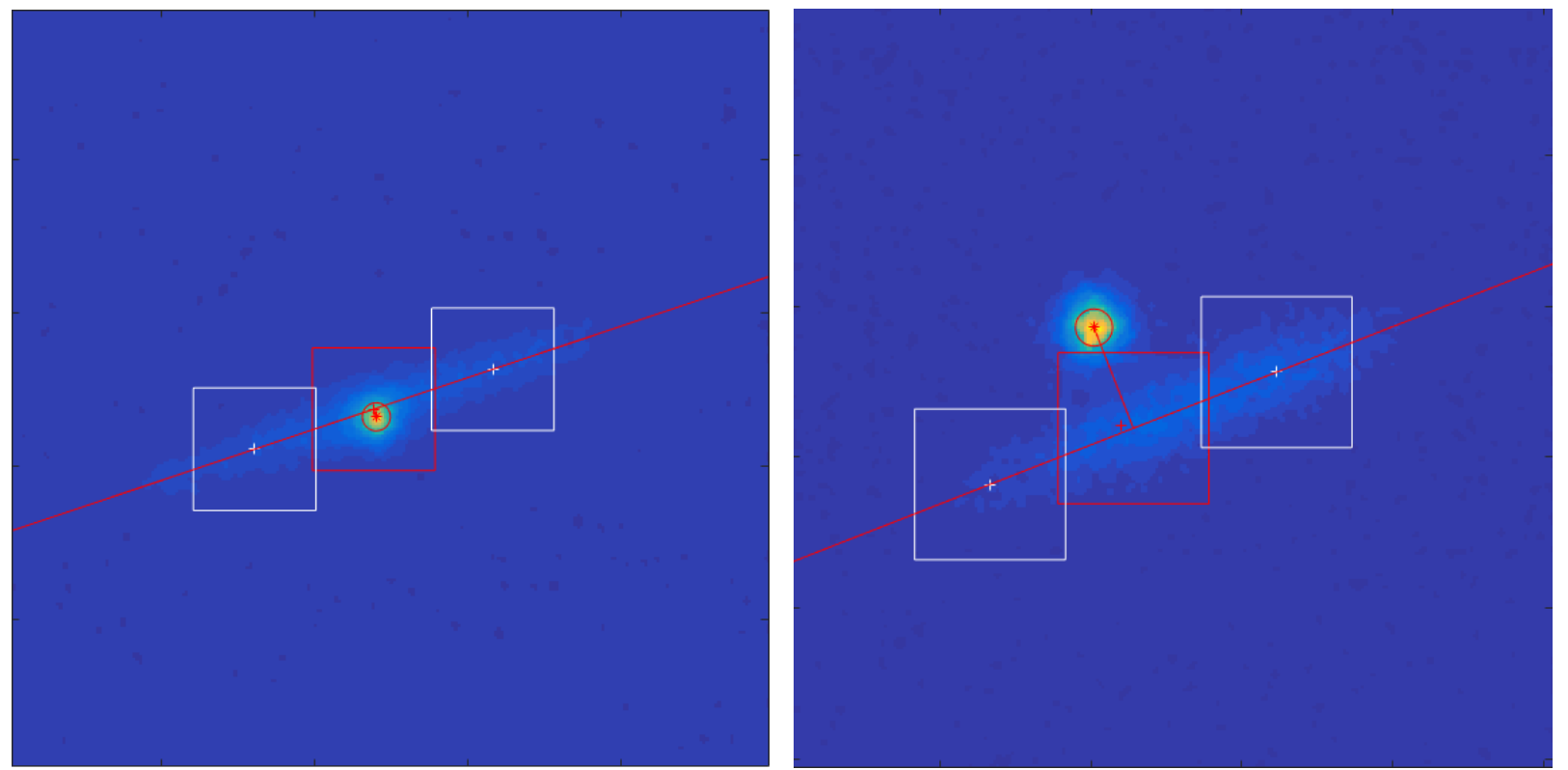

Figure 5. Typical scenario of the scene for a NGS and elongated LGS, as seen from the ESA-OGS telescope. Left: LGS and NGS are overlapped. Right: LGS and NGS are separated by few arcseconds.

After performing all the image cleaning steps, the center of gravity (CoG) of the NGS and LGS are estimated. For the NGS a 2D Gaussian fit is used to determine its CoG, while for the LGS the method of moments was used ${ }^{20}$. The LGS CoG was always estimated over the point projected by the NGS over the LGS major axis. Figure 5 presents an example of two typical situations for the CoG estimation. The left image shows the case when the NGS and the LGS are overlapped, for which the LGS CoG is estimated by taking the mean of the CoG position of two white boxes at each side of the NGS that lay over the LGS major axis. The right image shows the case where the NGS and LGS are separated, for which the LGS CoG is directly estimated over the point projected by the NGS over the LGS major axis.

\section{PRELIMINARY RESULTS}

After the image processing, a preliminary analysis of the measurements has been performed. In this paper, only the data analysis for the sequence of HIP61385 is presented. The analysis work is in progress. First the tilt of the LGS and the NGS are subtracted and the variance is calculated with a sliding window of 1000 samples. An average over 1 rrad of angular separation between both stars is done for each of the parameters plot in this section.
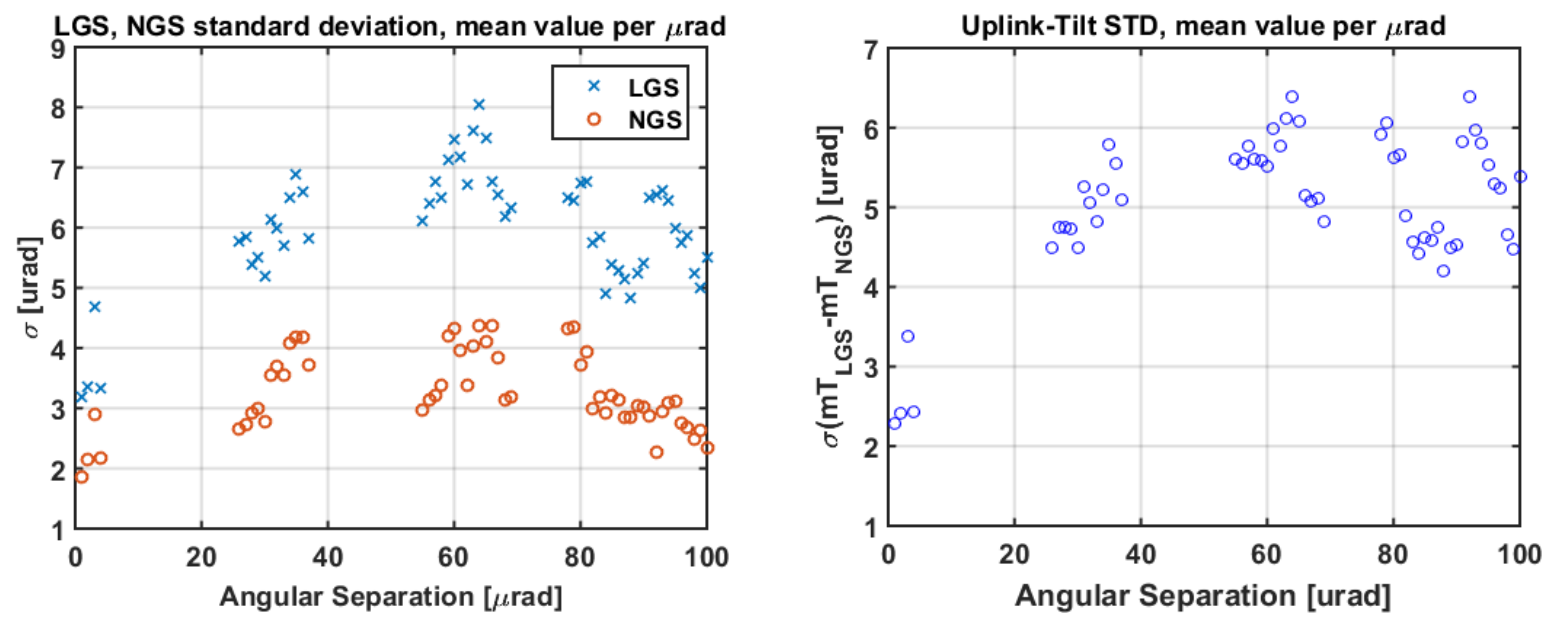

Figure 6. Left: standard deviation of the LGS and NGS tilts versus their angular separation. Right: standard deviation of the subtraction between LGS and NGS tilts, showing subarcsecond values $(4.85 \mu \mathrm{rad}=1 \mathrm{arcsec})$ 
The standard deviation of the measured LGS and NGS tilts are shown in Figure 6 (left) versus the angular separation between both stars. Thirty-eight sequences were recorded with HIP61385, taking around two hours from 21:00 to 23:00, between first and last frame. That corresponds to around 1 minute per $\mu$ rad. The observation elevation angle ranged between $70^{\circ}$ and $50^{\circ}$. In Figure 6 (left) the change in the turbulence conditions can be seen along the star separation sequence as a trend and also in smaller time scale with fluctuations of the standard deviation of the tilt.

The right plot of Figure 6 shows the standard deviation of the difference between the LGS and NGS measured tilts. Following equation (2), the estimation of the uplink-tilt can be done for small separations of the LGS with respect to the NGS. Therefore the standard deviation of the uplink-tilt, the beam-wander, is estimated to be between $2 \mu \mathrm{rad}$ and $3 \mu \mathrm{rad}$ at the beginning of this sequence, which is in the same order of magnitude than the uplink-tilt calculated by the theoretical models in Figure 1 (left). For larger separations, the standard deviation of the uplink-tilt increases, which can be due to the decrease of correlation or to the increase of the turbulence conditions as shown in left plot of Figure 6.

To normalize the effect of the turbulence, the ratio between the variance of the tilt-subtraction and the variance of the LGS-tilt is shown in Figure 7 (left). The ratio is around 0.5 for small angular star separations, approaching to 1 for an angular star separation in the order of $100 \mu \mathrm{rad}$. Assuming the LGS and NGS downlink tilts perfectly correlated for small separations, the ratio of 0.5 let us estimate the downlink contribution to the LGS tilt variance as half of the total LGS tilt variance. This last assumption allows finding a reasonable value to $\operatorname{std}\left(T_{L G S}^{d}\right)$ in equation (2) in order to be able to estimate the correlation between both LGS and NGS downlink paths. In the right plot of Figure 7, the correlation between the NGS and LGS downlink paths $\rho_{L_{G S}, N G S}$ is shown. As expected the trend of the correlation is to decrease with increasing angular separation. Fluctuations in the correlation can be clearly seen, due to changing turbulence conditions in smaller time-scale than the general trend in time, as already observed in the other figures.

Three curve fitting have been performed with a Gaussian function with three difference standard deviations in Figure 7 (right). Data points, shown as circles in this figure, represent the average value of the correlation as a function of the angular separation between the LGS and NGS. Taking this data the fitted standard deviation is 90 rrad. Moreover taking the $\pm 1 \sigma$ points for each $\mu \mathrm{rad}$, one can estimate a best case and a worst case with standard deviations of $117 \mu$ rad and $76 \mu \mathrm{rad}$, respectively. Taking only the data up to $40 \mu \mathrm{rad}$ another fitting estimates a standard deviation of $45 \mu \mathrm{rad}$.
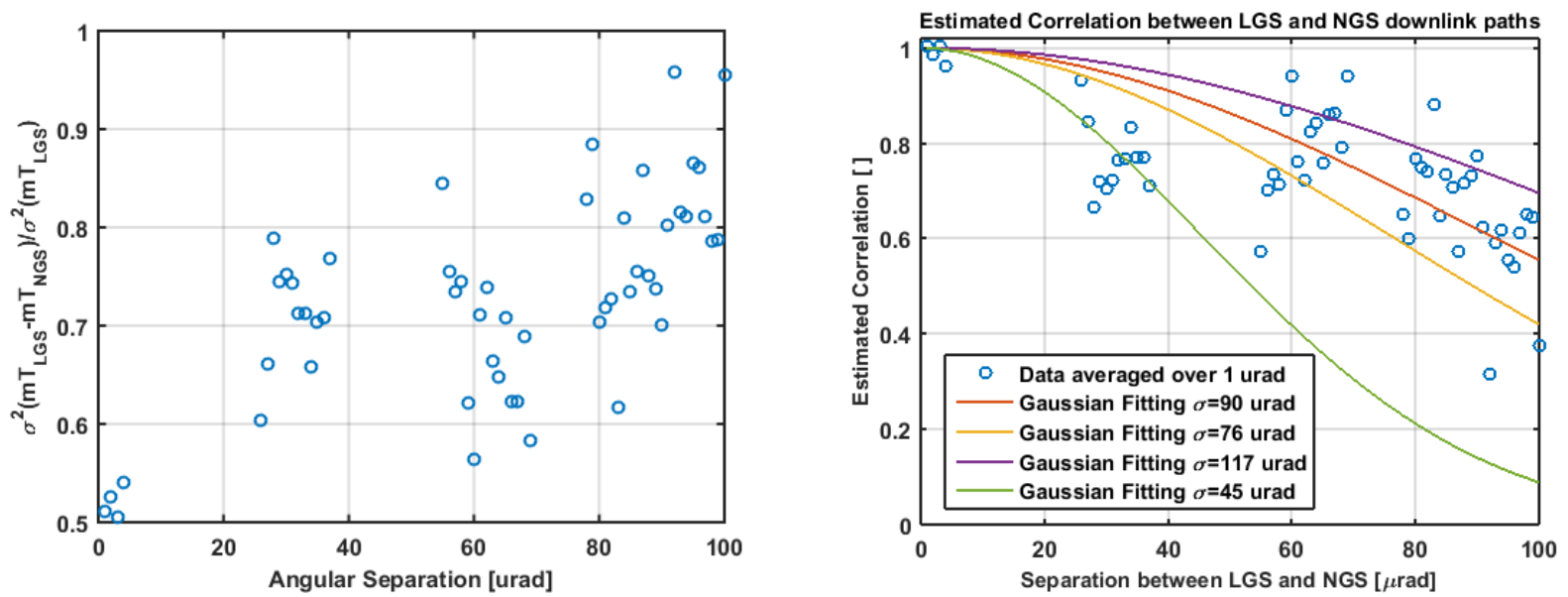

Figure 7. Left: ratio of tilt-subtraction variance over LGS-tilt variance. Right: estimated correlation between LGS and NGS downlink paths.

\section{CONCLUSIONS}

Laser guide stars may offer a solution for pre-compensating the uplink turbulence effects by means of adaptive optics. In scenarios where a downlink signal from the space-craft is too weak or the point-ahead angle is too large, laser guide stars provides a reference signal in the direction of the uplink link propagation. Moreover, the possibility to derive the 
atmospheric tip-tilt from LGS is of great interest also for the astronomical observatories. Although possible methods have been proposed, no systematic experiments have been conducted. The retrieval of the atmospheric turbulenceinduced tip-tilt has large application interest; hence this research is of large interest for DLR, ESA and ESO programs.

In this paper two measurement campaigns are presented, aimed at starting the research activities. They took place in April and July 2016 thanks to collaboration between DLR and ESO with the support of ESA. The goal was to make field tests to validate the theoretical correlations expected for the LGS and NGS tilt induced by the atmospheric turbulence, and to test the related hardware needed. We have obtained the goals.

The theoretical basis, the measurement campaign equipment and the first results have been described. The uplink-tilt or beam-wander could be measured by using the laser guide star and a natural star. The estimated values fall between 2 and $3 \mu \mathrm{rad}$, which is in line with one of the theoretical models. The correlation between two downlink paths with respect to the star separation could be characterized versus the angular separation between both stars. The first results show that the decay of $3 \mathrm{~dB}$ in the uplink-tilt correlation happens at larger angular separation than expected: after 50 to $100 \mu \mathrm{rad}$.

Further work will be performed to analyze the measured data, and to investigate methods to separate in real time the turbulence-induced tilt, between the uplink and the downlink. This is toward both, astronomical adaptive optics with $100 \%$ sky coverage and the use of laser guide stars in the ground-to-space communications. Experimental verification of the proposed methods will follow, pending approval of a proposal for collaboration on this R\&D between the participating institutes.

We would like to thank IAC for their support during the measurements campaign.

These measurement campaigns were performed under ESA contract 4000118061/16/D/MB and under the ESO Technology Development Program on Laser Guide Stars System R\&D.

\section{REFERENCES}

[1] Bonaccini Calia, D, Hackenberg, W, Holzlöhner, R, Lewis, S \& Pfrommer, T The Four-Laser Guide Star Facility - Design considerations and system implementation. Advanced Optical Technologies 3, 345-361 (2014).

[2] Holzlöhner, R et al. Comparison between observation and simulation of sodium LGS return flux with a 20W CW laser on Tenerife. Proc. SPIE 9909, 99095E-99095E-18 (2016).

[3] Bardou, L et al. Getting ready for the first on sky experiment using an ELT-scaled elongated sodium laser guide star. Proc. SPIE 9909, 990944-990944-11 (2016).

[4] Bonaccini Calia, D et al. The ESO transportable LGS Unit for measurements of the LGS photon return and other experiments. Proc. SPIE 8450, 84501R-84501R-12 (2012).

[5] Tolker-Nielsen, T \& Guillen, J-C SILEX The First European Optical Communication Terminal in Orbit. ESA bulletin 96, (1998).

[6] Gütlich, B, Meyer, R, Philipp-May, S \& Pagels-Kerp, A German Roadmap on Optical Communication in Space. Advanced Solid-State Lasers Congress LM1B.2 (2013).doi:10.1364/LSC.2013.LM1B.2

[7] Mata Calvo, R et al. Transmitter diversity verification on ARTEMIS geostationary satellite. Proc. SPIE 8971, 897104-897104-14 (2014).

[8] Romba, J, Sodnik, Z, Reyes, M, Alonso, A \& Bird, A ESA’s bidirectional space-to-ground laser communication experiments. Proc. SPIE 5550, 287-298 (2004).

[9] Moll, F \& Knapek, M Free-space laser communications for satellite downlinks - Measurements of the atmospheric channel. 62nd International Astronautical Congress (2011).

[10] Boroson, D M et al. Overview and results of the Lunar Laser Communication Demonstration. Proc. SPIE 8971, 89710S-89710S-11 (2014).

[11] Andrews, L \& Phillips, R Laser Beam Propagation through Random Media. (SPIE Press: 2005).

[12] Sasiela, R J Electromagnetic Wave Propagation in Turbulence. (SPIE: 2007).

[13] Reyes, M et al. Artemis Laser Link - Final Report. (Instituto de Astrofísica de Canarias: 2004).

[14] Esposito, S, Ragazzoni, R \& Riccardi, A Focus anisoplanatism effects on tip-tilt compensation for adaptive optics with use of a sodium laser beacon as a tracking reference. J. Opt. Soc. Am. A 13, 1916-1923 (1996).

[15] Ragazzoni, R Robust tilt determination from Laser Guide Stars using a combination of different techniques. aap 319, L9-L12 (1997).

Copyright 2017 Society of Photo-Optical Instrumentation Engineers. This paper was published in SPIE Photonics West 2017 and is made available as an electronic reprint with permission of SPIE. One print or electronic copy may be made for personal use only. Systematic reproduction and distribution, duplication of any material in this paper for a fee or for commercial purposes, or modification of the content of the paper are prohibited. 
[16] Castro-Almazán, J A et al. The bistatic geometry for Na profiling with LGS at Teide Observatory. Proc. SPIE 9909, 99093M-99093M-6 (2016).

[17] Troendle, D C et al. ALPHASAT TDP1 - Three Years Optical GEO Data Relay Operations. 34th AIAA International Communications Satellite Systems Conference (2016).

[18] Bonaccini Calia, D et al. ELT LGS-AO: Optimizing the LGS Return Flux. Adaptive Optics for Extremely Large Telescopes II (2011).

[19] Foy, R \& Foy, F C Optics in Astrophysics. Proceedings of the NATO Advanced Study Institute on Optics in Astrophysics, Cargèse, France from 16 to 28 September 2002, (Springer: 2005).

[20] Stone, R C A comparison of digital centering algorithms. Astronomical Journal 97, 1227-1237 (1989). 